\title{
Mycobacteriophages: An important tool for the diagnosis of Mycobacterium tuberculosis (Review)
}

\author{
XIAOYAN FU ${ }^{1}$, MINGXING DING ${ }^{1,2}$, NING ZHANG ${ }^{1}$ and JICHENG LI \\ ${ }^{1}$ Department of Medical Sciences, Jinhua College of Profession and Technology, Jinhua, Zhejiang 321007; \\ ${ }^{2}$ Institute of Cell Biology, Zhejiang University School of Medicine, Hangzhou, Zhejiang 310058, P.R. China
}

Received May 26, 2014; Accepted February 13, 2015

DOI: $10.3892 / \mathrm{mmr} .2015 .3440$

\begin{abstract}
The prevention and control of tuberculosis (TB) on a global scale has become increasingly important with the emergence of multidrug-resistant TB. Mycobacterium tuberculosis phages have been identified as an important investigative tool. Phage genomes exhibit a significant level of diversity and mosaic genome architecture, however, they are simple structures, which are amenable to genetic manipulation. Based on these characteristics, the phages may be used to construct a shuttle plasmid, which is an indispensable tool in the investigation of TB. Furthermore, they may be used for rapid diagnosis and assessing drug susceptibility of TB, including phage amplified assessment and reporter phage technology. With an improved understanding of mycobacteriophages, further clarification of the pathogenesis of TB, and of the implications for its diagnosis and therapy, may be elucidated.
\end{abstract}

\section{Contents}

1. Introduction

2. Molecular tools of Mycobacterium investigation

3. TB diagnosis and drug sensitivity assessments based on mycobacteriophages

4. Conclusion

\section{Introduction}

The emergence of multi-drug resistant tuberculosis (TB) has increased focus on the global prevention and control of TB. At present, TB control faces a number of challenges, including low sensitivity, poor specificity and treatment complications, long detection cycles of traditional diagnostic techniques

Correspondence to: Mrs. Xiaoyan Fu, Department of Medical Sciences, Jinhua College of Profession and Technology, 1188 Wuzhou Street, Jinhua, Zhejiang 321007, P.R. China

E-mail: 1012005114@qq.com

Key words: mycobacteriophage, Mycobacterium tuberculosis and a decline in the immune function of traditional vaccines, including Bacillus Calmette-Guerin (1). Since 1947, when mycobacteriophages were first isolated and identified by Gardner et al (2), >3,680 types of mycobacteriophage have been isolated from different sources, of which $>544$ types of mycobacteriophages have undergone complete genome sequencing (3). As a member of the bacteriophage family, which are DNA viruses, mycobacteriophages are able to infect the host Mycobacteria specifically. L5 (4), D29 (5) and TM4 (6) are the mycobacteriophages, were the earliest to undergo genome sequencing and are the most widely used in the investigation of TB. Greater understanding of the structure and function of the mycobacteriophage genome has increased awareness of the importance of investigating the diagnosis and treatment of Mycobacterium tuberculosis.

\section{Molecular tools of Mycobacterium investigation}

In 1964, Tokunaga and Sellers (7) were the first to use the D29 phage to successfully induce outer DNA into M. smegmatis, which demonstrated the feasibility of mycobacteriophage DNA transfection. Subsequently, in 1970, I3 was successfully enriched with the use of $M$. smegmatis by Raj and Ramakrishnan (8), which again supported the viability of transduction. Since these early experiments, the rapid development of genetic engineering has led to the construction of a number of high-efficiency cloning and expression vectors. Recombinant DNA technology has also progressed, however, due to a lack of understanding of the mycobacteriophage genome, progress in recombinant DNA technology for the application of mycobacteria has been slow. Mycobacteria have a lipid-rich cell wall, which limits the ability of the exogenous DNA to pass through the cell wall and, therefore, foreign DNA are unable to be stably integrated and expressed in mycobacteria (9).

Jacobs et al (10) succeeded in creating a method of mycobacterial genome transfer in 1987, overcoming the difficulties in investigating mycobacterial genes. The plasmid DNA of Escherichia coli was inserted into the non-essential region of the TM4 genome, to successfully construct a recombinant shuttle plasmid vector (Fig. 1). The vector was a dual function shuttle plasmid vector, which was not only a plasmid replicated in the $E$. coli, but also a phage replicated within 
the mycobacteria. Therefore, this overcame the deficiencies of traditional plasmid vectors, carrying a limited length of exogenous DNA fragments, and the insufficient capacity of bacterial transformation. The experimental results demonstrated that the recombinant shuttle vector was transfected into fast-growing M. smegmatis, however, this experiment was not successful in slow growing mycobacteria, including Bacillus Calmette-Guérin (BCG) strains and M. tuberculosis. Despite this, it demonstrated that recombinant shuttle plasmids may eventually be suitable for use to induce exogenous DNA into the BCG vaccine strains to develop a recombinant mycobacterial vaccine. Snapper et al (11) also constructed a shuttle plasmid successfully based on L1 and demonstrated the stable insertion and replication of exogenous DNA in M. smegmatis. Lee et al (12) achieved an effective and stable transformation using the mild site-specific integrated L5 mycobacteriophage. These findings demonstrated the building of an efficient integration vector by integrating the plasmid sequences into the mycobacterial genome, with effective integration of the TB mycobacterium and BCG to obtain stable recombinant DNA.

These previous studies demonstrated that the shuttle plasmid was of value for specific transduction (13), transposon transfer $(14,15)$ and the introduction of diagnostic reporter genes $(16,17)$. The development of this vector system promotes the genetic analysis Mycobacterium pathogens and the development of a recombinant vaccine.

\section{TB diagnosis and drug sensitivity assessments based on mycobacteriophages}

TB is the most important global public health problem at present. In 2010, there were 8.8 million incident cases of TB, 1.1 million deaths from TB among HIV-negative people, and an additional 0.35 million deaths from HIV-associated TB (18). Therefore, the control of the condition via rapid and accurate TB diagnosis is important. The demand for a simple, fast, safe, sensitive and accurate $M$. tuberculosis antibiotic susceptibility assessment has become increasingly urgent, as a result of the emergence and spread of multidrug-resistant TB and extensively drug-resistant tuberculosis (XDR-TB). In previous years, molecular techniques for the diagnosis of TB have been rapidly developed. The nucleic acid amplification method, involving nucleic acid probes, polymerase chain reaction, DNA sequencing, Gene Chip and Xpert MTB/RIF enables rapid diagnosis and assessment of resistance of M. tuberculosis (19). Although the majority of the techniques are fast with a high sensitivity, the requirement for specialized instruments and high costs significantly limited its dissemination and application in the majority of countries with a high burden of TB. In addition, there was a $\$ 1$ billion gap in the funds of the World Health Organization for TB management and control in 2012, causing financial pressure in the diagnosis and treatment of TB (18). Assessments, which enable the rapid detection of mycobacteriophages have numerous advantages, including high speed, simplicity, specificity, security, no requirement for specialist equipment and lower costs, and they enable the quantitative detection of viable cells. Therefore, mycobacteriophages have become an ideal tool for TB diagnosis and assessment of drug susceptibility.
Phage amplification technology. The investigation of phage amplification technology can be traced back to 1965. A study by Sellers et al (20) observed the effects of anti-TB drugs on mycobacteriophages. The results of the experiments demonstrated that streptomycin (STR) was able to prevent the phages copying in M. smegmatis, whilst not affecting the phage replication of the progeny in resistant strains, or their subsequent release. Since this observation, other drugs, including clofazimine, colistin, rifampicin (RIF) and STR have also been assessed for their effects on the synthesis of D29 $(21,22)$. In these studies, D29 was able to affect slow-growing pathogenic mycobacteria and the fast-growing environmental strains, and visible plaque formed in the fast-growing $M$. smegmatis bacteria following overnight incubation. The existence of viable bacteria can be determined by rapid detection of the release of progeny phages following infection of the mycobacterium target using this technique. These experiments laid the foundation for the subsequent development of phage amplification technology and its application in assessing anti-mycobacterial drug sensitivity.

The phage amplification technology, which is in current clinical use was first described by Wilson et al (23) in 1997, and further defined on the basis of further modifications by McNerney et al (24). Subsequently, Biotec Laboratories Ltd. (Ipswich, UK) developed corresponding commercial kits, FASTPlaque $\mathrm{TB}^{\mathrm{TM}}$ and FASTPlaqueTB-MDRi ${ }^{\mathrm{TM}}$, or FASTPlaqueTB-RIF ${ }^{\mathrm{TM}}(25,26)$, which were used for the rapid detection of $M$. tuberculosis and for the assessment of multi-drug resistance (Fig. 2). Firstly, D29 phages were introduced into $M$. tuberculosis, in which they reproduced. The phages, which did not enter the cell were killed by virucide agents, however, the phages that entered the viable $M$. tuberculosis were not affected. The phages lysed the bacteria in vivo following replication in the bacteria. Subsequently, the releasing phages infected and lysed $M$. smegmatis to form plaques. As this assessment is reliant on the presence or absence of plaques to determine the result, this method generally requires 1-2 days to produce results. As there is a proportional association between the quantity of plaques and the quantity of $M$. tuberculosis in the specimens, the content of $M$. tuberculosis in the sample can be calculated according to the number of plaques. As M. tuberculosis is cleaved during the experiment, the experiment has fewer safety concerns for the individuals involved.

The clinical effects of this assay have been evaluated in several countries, including Egypt (27), Pakistan (28), South Africa (29) and Spain (30). Kalantri et al (31) performed a meta-analysis of the detection of $M$. tuberculosis in clinical samples, based on phage amplification technology in 2005 by examining the literature from databases, including Medline, EMBASE (http://www.elsevier.com/online-tools/embase), Web of Science (http://wok.mimas.ac.uk/) and BIOSIS Previews (http://biosispreviews.isihost.com/). A total of 13 studies were included, which complied with designated standards. The specificity and sensitivity of these assays were between 0.83 and 1.00, and between 0.21 and 0.94 , respectively, with sputum culture as a reference standard. The results revealed that the assay had a high specificity and a moderate/variable sensitivity, which required improvement. The predominant reasons for the lower sensitivity included anti-TB treatment prior to the experiment, 


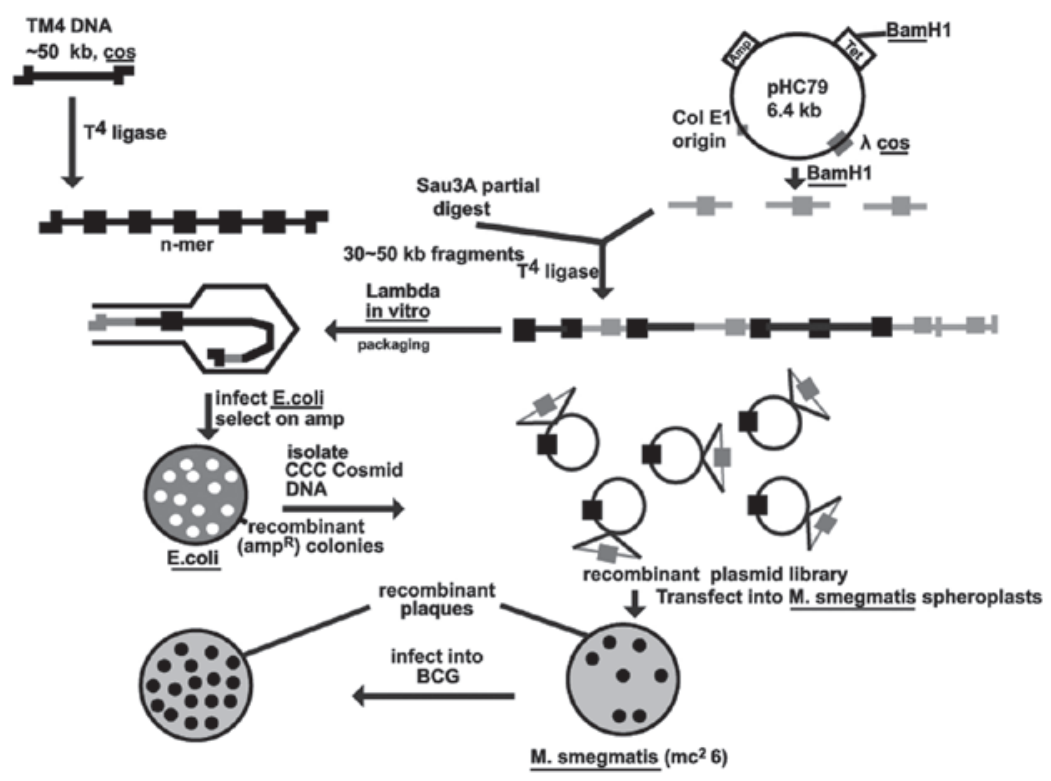

Figure 1. Diagram of the stages of construction of a shuttle plasmid. TM4 phage DNA is ligated and then partially digested with Sau3A to obtain fragments between 30 and $50 \mathrm{~kb}$. These DNA fragments are then ligated to the cosmid pHC79, which has been cleaved with BamHI. The ligation mixture is packed into the phage $\lambda$ head in vitro. The resulting phages are transduced into E. coli. The clones containing recombinant pHC79::TM4 DNA molecules are selected on media containing ampicillin. The plasmid is isolated from the pool of clones and then transfected into M. smegmatis to select for the shuttle plasmid, TM4 phages containing pHC79. The shuttle plasmid is able to infect and replicate in BCG vaccine strains. BCG, Bacillus Calmette-Guerin.
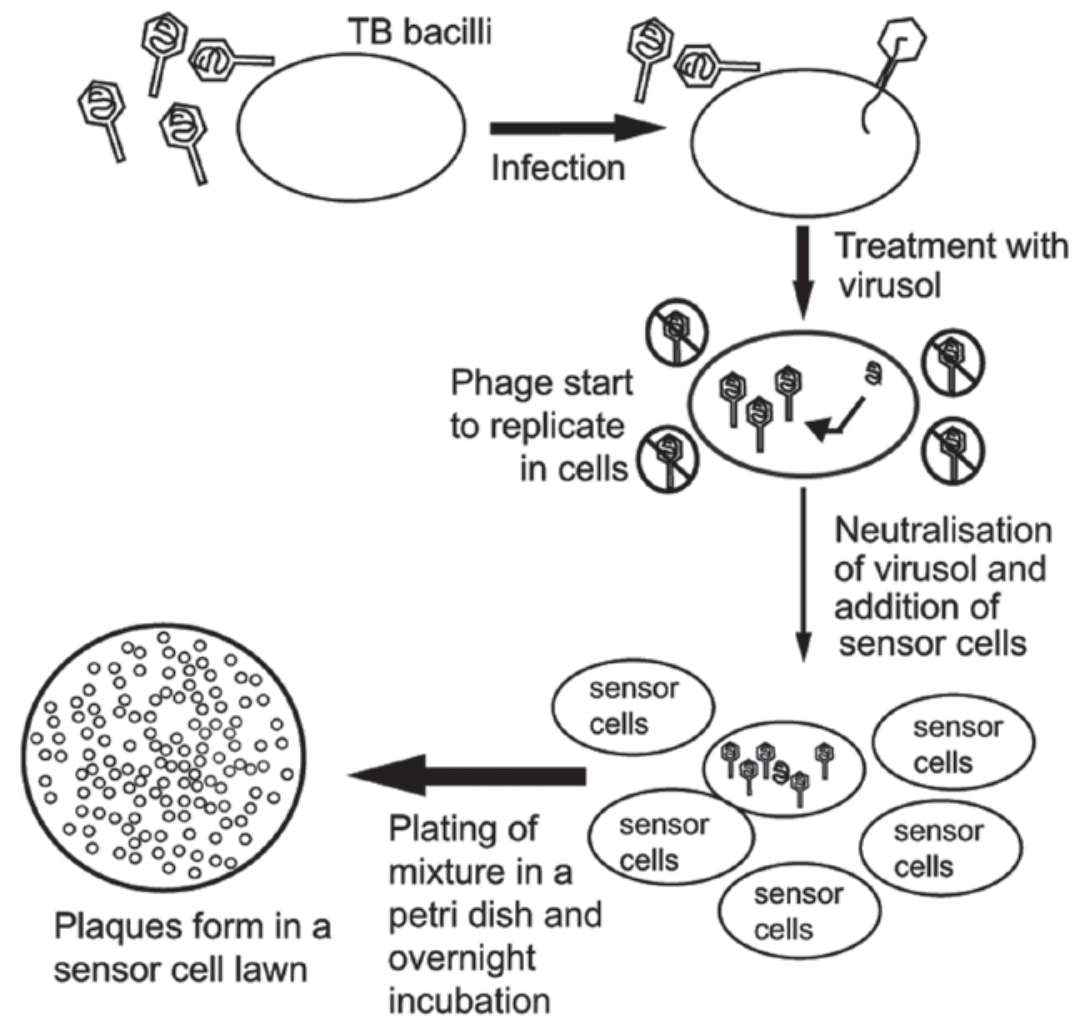

Figure 2. Diagrammatic representation of phage amplification technology. Mycobacteriophages infect TB bacilli in the sample. Following infection, the sample is treated with a potent virucide to destroy the mycobacteriophages that have not infected host cells. The remaining phages within the host organisms undergo a replication cycle, resulting in the release of the phages from the host cells. The released phages are allowed to infect a lawn of rapidly-growing, non-pathogenic mycobacteria (sensor cells). Clearing plaques indicate the presence of TB cells. TB, tuberculosis.

sample transportation, environmental conditions and the selection of detergents. Therefore, further investigations are required to improve the sensitivity of the phage-based assessment.
The Foundation for Innovative New Diagnostics (FIND) extensively evaluated the role of the FASTPlaque assessment technique in rifampin resistance in 2007 (32). The FASTPlaque 
assay failed to achieve the desired objectives in two trial sites in South Africa. Therefore, FIND terminated the FASTPlaque assessment pilot program until a satisfactory improvement had been made (32). Therefore, although FASTPlaque assessment can be widely used for the rapid diagnosis of TB, however, further improvement of the optimization techniques is required.

Luciferase reporter phage. The fluorescent reporter phage is a rapid detection system for $M$. tuberculosis susceptibility and drug susceptibility based on recombinant DNA technology. The first generation of luciferase reporter phages (LRPs) were developed successfully by Jacobs et al in 1993 (16). These were constructed from the phAE39 plasmid shuttle, on the basis of TM4, and the firefly luciferase (FFlux) gene was inserted using a potent promoter of heat shock protein 60 (hsp60; Fig. 3). LRPs are able to transfer recombinant DNA into mycobacteria, including the $M$. smegmatis and $M$. tuberculosis BCG vaccine strains, In the presence of adenosine triphosphate and luciferin, FFlux is able to continuously express and generate an optical signal following mycobacterial infection. If there are at least $10^{4} /$ milliliter of $M$. tuberculosis in the sample, the relative light units can be detected within a few minutes following LRP infection of the live mycobacteria. This method reduced the reporting duration considerably compared with the traditional detection methods. LRPs based on L5 (33) and D29 (34) have been subsequently constructed, however, various defects remain. The mild L5 mycobacteriophage is unable to infect the M. tuberculosis complex, which limits its application in the drug resistance detection of clinical samples. The lytic characteristics of D29 and TM4 result in the loss of light output and reduced sensitivity. Since the characteristics of lytic phages may reduce light output, Kumar et al (35) constructed new LRPs using the mild Che12 bacteriophage to increase light output and improve the sensitivity of the assessment. Carriere et al (36) addressed the problem using a number of strategies, including changing the position of FFlux in the phage genome, isolating host-range mutant phages and inducing temperature-sensitive mutants of phages to screen more sensitive mutants compared with the first generation LRPs. Although the sensitivity of LRPs has improved, these LRPs can infect mycobacteria with the exception of M. tuberculosis, leading to misdiagnosis in clinical practice, therefore, it is necessary to improve the experimental program to confirm the presence of the M. tuberculosis complex. Considering these problems, Riska et al (37) added $\varrho$-nitro- $\alpha$-acetylamino- $\beta$-hydroxy propiophenone to the substrate to selectively inhibit the $M$. tuberculosis complex bacteria, and combined the corrected program with the ordinary LRPs to accurately distinguish strains of the M. tuberculosis complex and non-TB mycobacteria, which improved the accuracy of the anti-TB drug susceptibility assessment.

As the phages only replicate in living cells, the limitations of the above methods include the ability to detect only viable cells in the sample. However, M. tuberculosis is dormant in the bodies of numerous patients with clinically latent infections (38), presenting a challenge in detecting dormant M. tuberculosis. Dusthackeer (39) used the hsp60,

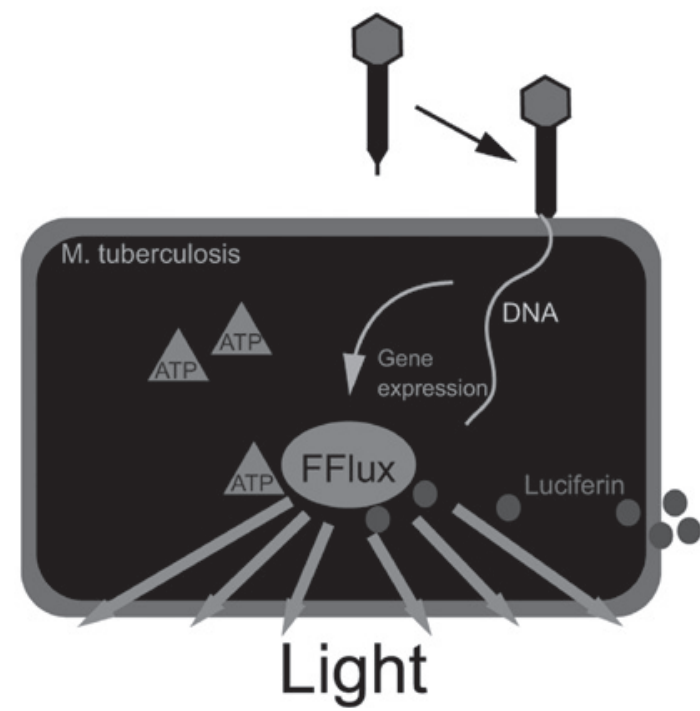

Figure 3. Schematic representation of the luciferase reporter phages. The mycobacteriophage, with a firefly luciferase gene ( $f f u x)$ inserted within its genome, is used to infect M. tuberculosis within a sample. In addition to the enzyme, light output requires two substrates, luciferin and ATP. Luciferin is added exogenously and adsorbed rapidly by the M. tuberculosis cells. ATP is provided by the viable host cells. Therefore, following infection of the viable target cells, the fflux gene is expressed and light is produced. ATP, adenosine triphosphate.

isocitrate lyase and $\alpha$ crystal protein ( $\alpha$-crystallin) gene promoters to promote the gene expression of FFlux, and successfully detected the dormant M. tuberculosis bacteria. Dusthackeer et al (40) improved the experimental method further by detecting the sputum samples without the primary culture. It was suggested that this provided a better simulation of the natural state of dormant bacteria. The results of this study supported this hypothesis, which demonstrated the possibility of potential TB detection.

Banaiee et al (41) compared the assessment of the drug susceptibility of LRPs with the BACTEC 460 assay as a reference in clinical applications. The BACTEC 460 assay is a semi-automated phage-based antibiotic susceptibility assay. The results revealed that the diagnostic accuracy of LRPs reached $98.4 \%$, and the drug detection accuracy rate was $100 \%$. The sensitivity and specificity for the detection of RIF drug resistance were $100 \%$, and for isoniazid (INH) were 100 and $97.7 \%$, respectively. The duration required to perform an LRP trial was considerably reduced, just 2 days, compared with the BACTEC 460 assessment, which required 9 days. In addition, its economic cost is low, at $\$ 0.40$ for each strain. This semi-automated LRP assessment technique is ideal for laboratories with limited funds, enabling assessments in economically underdeveloped countries experiencing a high burden of TB. Minion and Pai (42) performed a meta-analysis of the phage-based assessments of RIF resistance prior to 2009, a total of 31 studies were included in a sample of 3,085 studies, and the phage amplified biological assessment and LRP assessment were compared. The results revealed that the sensitivity and specificity of the LRPs were 99.3 and $98.6 \%$, marginally higher than the phage amplified biologically assessment at 98.5 and $97.5 \%$. However, a similar investigation with a larger LRP sample size is required. 


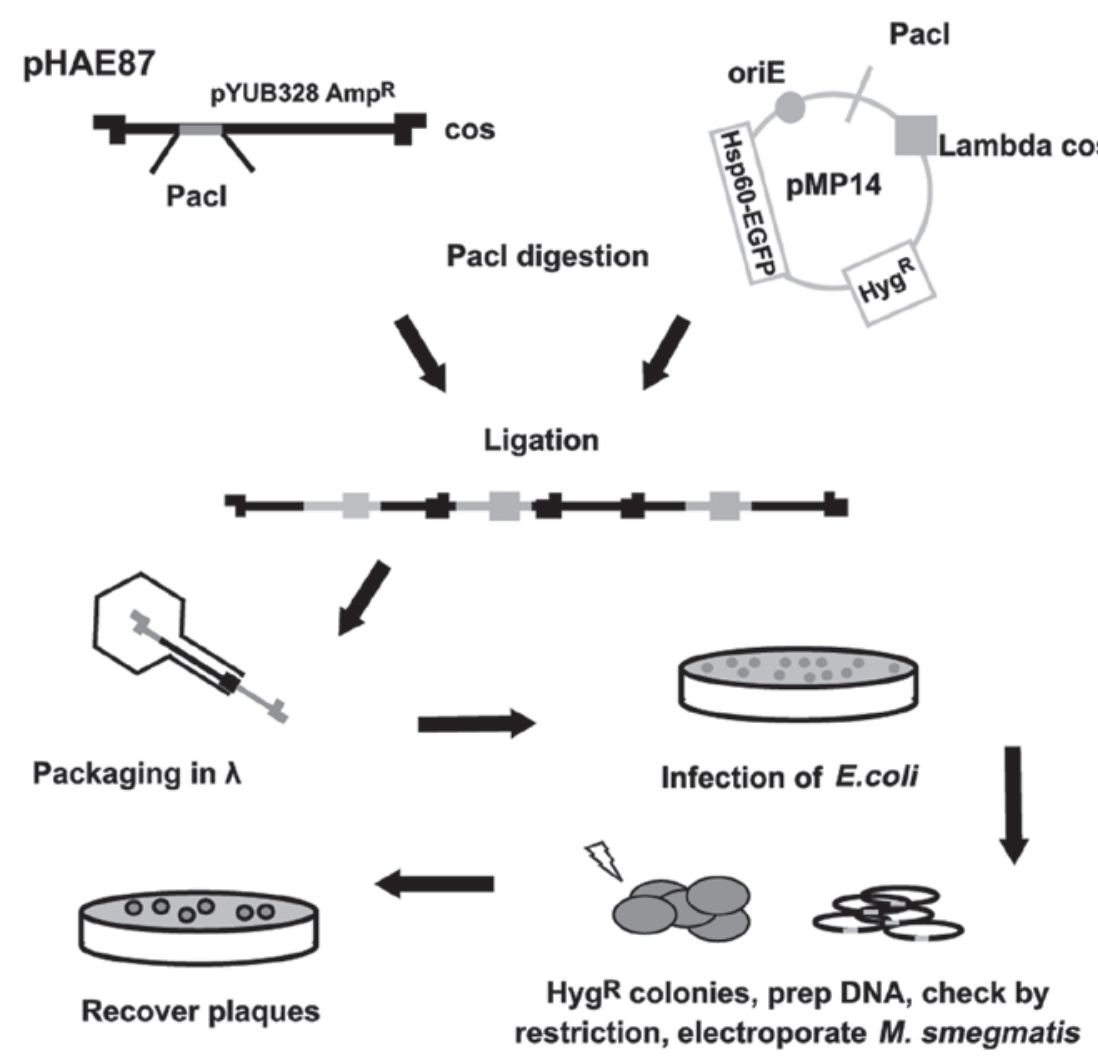

Figure 4. Fluoromycobacteriophage construction. Schematic representation of phAE87::hsp60-EGFP construction. The phAE87 huttle plasmid is a conditionally replicating derivative of phage TM4, in which the cosmid moiety is flanked by PacI restriction sites. A plasmid derivative of pYUB854, containing the EGFP gene (pMP14), is used to replace the cosmid in phAE87. This is followed by $\lambda$ packaging and recovery in E. coli. EGFP, enhanced green fluorescent protein; $\mathrm{Hyg}^{\mathrm{R}}$, hygromycin-resistance marker; $\mathrm{Amp}^{\mathrm{R}}$, ampicillin-resistance marker.

Fluoromycobacteriophages. Fluoromycobacteriophages, a novel phage, were identified in 2009 , and differ from the previously reported LRPs. Piuri et al (17) constructedthe fluoromycobacteriophages phAE87::hsp60-EGFP and phAE87::hsp60-ZsYellow (Fig. 4). Green fluorescent protein $(G F P)$ or the ZsYellow fluorescent markers were introduced into M. tuberculosis, to detect the drug susceptibility using fluorescence microscopy or flow cytometry within $24 \mathrm{~h}$. The technique has several advantages compared with the LRPs, as no substrate is required, $<100 / \mathrm{ml} \mathrm{M}$. tuberculosis can be identified, drug-resistant strains may be detected in the mixed population and the biosecurity of the samples is enhanced by polyformalin-fixed processing. Rondón et al (43) also designed an enhanced GRP (EGFP) phage, phAE87:: hsp60-EGFP, containing EGFP on the basis of TM4. This technique was used to detect drug resistance of M. tuberculosis strains to INH, RIF and STR, and the results revealed that the sensitivity of this technique to all antibiotics was $94 \%$, and the specificities of INH, RIF and STR were 90, 93 and 95\%, respectively, compared with the resazurin microplate technique. The results of the resazurin microplate assay also exhbibited $94 \%$ sensitivity for INH and RIF, whereas sensitivity for STR was higher at $98 \%$. The reporting time-period of this technique was 2-3 days and the costs were $\sim \$ 2$. Although $E G F P$ phage technology for rapid screening of combined drug resistance is of potential economic value, it requires further simplification to suit clinical requirements as a rapid and economic way to detect multidrug-resistant or extensively drug-resistant strains of
TB in resource-poor settings with minimal infrastructure, and improve sensitivity.

One problem of fluoromycobacteriophages is that, as a potent mycobacteriophage, TM4 initially infects bacteria, and then cleaves it, terminating the expression of EGFP. Therefore, the sensitivity of fluoromycobacteriophages is reduced as the duration of EGFP expression is shortened (43). To address this problem using bacteriophage recombineering of electroporated DNA, da Silva et al (44) inserted a $\mathrm{P}_{h s p 60^{-}} e g f p$ cassette into the $\mathrm{D} 29$ mycobacteriophage genome to construct a novel reporter phage. Based on the this novel reporter phage, an attempt was made to construct a lysis-defective mutant by deleting the lys A gene, however, it was not possible to purify the mutant. Despite this, the attempt provided a novel strategy for the development of a more sensitive reporter phage.

Another problem of fluoromycobacteriophages is that the adsorption of TM4 is relatively inefficient. However, mutants can be isolated with enhanced adsorption, which may provide a strategy for improving the efficiency of recovery. Piuri et al (45) constructed a plasmid expressing the major capsid protein gene (gp9) of TM4, and containing Strep-tag II (STAG II). Particles with capsids composed of wild-type and STAG-tagged subunit mixtures were able to grow to high titers, exhibited good infectivity and were suitable for used to isolate phage-bacterium complexes. Reporter phage technology based on the fluorescent protein emitting principle requires further evaluation of its clinical effects. 


\section{Conclusion}

Since mycobacteriophages were identified 50 years ago, $>2,439$ types of mycobacteriophages have been isolated and the genome sequences of $>363$ types of mycobacteriophages have been completed. Mycobacteriophage genomes have several features, including diversity and mosaicism, a simple structure and amenability to genetic manipulation. Based on these characteristics, a shuttle plasmid was constructed for TB investigation using recombinant DNA technology. With improvements in genomics, shuttle plasmids have also been used to build different luciferase reporter phages and fluoromycobacteriophages, which have contributed to the investigation of mycobacteria and TB. Following several years of limited studies, phage therapy is again an active area of investigation, particularly in bacteriophage lyase. As investigation into mycobacterial phages progresses, improvements in the current understanding of its role in TB, and particularly its diagnosis and treatment, is expected.

\section{References}

1. Weir RE, Gorak-Stolinska P, Floyd S, et al: Persistence of the immune response induced by BCG vaccination. BMC Infect Dis 8: 1-9, 2008.

2. Gardner GM and Weiser RS: A bacteriophage for Mycobacterium smegmatis. Proc Soc Exp Biol Med 66: 205, 1947.

3. Mycobacteriophage database. http://www.phagesdb.org/. Accessed 16 May, 2014

4. Hatfull GF and Sarkis GJ: DNA sequence: structure and gene expression of mycobacteriophage L5: a phage system for mycobacterial genetics. Mol Microbiol 7: 395-405, 1993.

5. Ford ME, Sarkis GJ, Belanger AE, et al: Genome structure of mycobacteriophage D29: implications for phage evolution. J Mol Biol 279: 143-164, 1998.

6. Ford ME, Stenstrom C, Hendrix RW, et al: Mycobacteriophage TM4: genome structure and gene expression. Tuber Lung Dis 79: 63-73, 1998.

7. Tokunaga T and Sellers M: Infection of Mycobacterium smegmatis with D29 phage DNA. J Exp Med 119: 139-149, 1964.

8. Raj CV and Ramakrishnan T: Transduction in Mycobacterium smegmatis. Nature 228: 280-281, 1970.

9. Jacobs WR Jr, Snapper SB, Tuckman M and Bloom BR: Mycobacteriophage vector systems. Rev Infect Dis 11: S404-S410, 1989.

10. Jacobs WR Jr, Tuckman M and Bloom BR: Introduction of foreign DNA into mycobacteria using a shuttle phasmid. Nature 327: 532-535, 1987.

11. Snapper SB, Lugosi L, Jekkelt A, et al: Lysogeny and transformation in mycobacteria: stable expression of foreign genes. Proc Natl Acad Sci USA 85: 6987-6991, 1988

12. Lee MH,Pascopella L, Jacobs WR Jr and Hatfull GF: Site-specific integration of mycobacteriophage L5: integration-proficient vectors for Mycobacterium smegmatis, Mycobacterium tuber culosis and bacille Calmette-Guérin. Proc Natl Acad Sci USA 88: 3111-3115, 1991

13. Bardarov S, Bardarov Jr S Jr, Pavelka Jr MS Jr, et al: Specialized transduction: an efficient method for generating marked and unmarked targeted gene disruptions in M.tuberculosis, M. bovis BCG and M. smegmatis. Microbiology 148: 3007-3017, 2002.

14. Bardarov S, Kriakov J, Carriere C, et al: Conditionally replicating mycobacteriophages: a system for transposon delivery to M. tuberculosis. Proc Natl Acad Sci USA 94: 10961-10966, 1997.

15. Sassetti CM, Boyd DH and Rubin EJ: Genes required for mycobacterial growth defined by high density mutagenesis. Mol Microbiol 48: 77-84, 2003.

16. Jacobs WR Jr, Barletta RG, Udani R, et al: Rapid assessment of drug susceptibilities of $M$. tuberculosis by means of luciferase reporter phages. Science 260: 819-822, 1993.

17. Piuri M, Jacobs WR Jr and Hatfull GF: Fluoromycobacteriophages for rapid, specific and sensitive antibiotic susceptibility testing of M. tuberculosis. PLoS One 4: e4870, 2009.
18. World Health Organization. Global tuberculosis control: WHO report 2011. WHO, Geneva, 2011.

19. Pholwat S, Ehdaie B, Foongladda S, Kelly K and Houpt E: Real-time PCR using mycobacteriophage DNA for rapid phenotypic drug susceptibility results for Mycobacterium tuberculosis. J Clin Microbiol 50: 754-761, 2012.

20. Tokunaga $\mathrm{T}$ and Sellers MI: Streptomycin induction of premature lysis of bacteriophage-infected mycobacteria. J Bacteriol 89: 537-538, 1965.

21. Phillips LM and Sellers MI: Effects of ethambutol, actinomycin D and mitomycin $\mathrm{C}$ on the biosynthesis of D29-infected mycobacterium smegmatis. In: Host-virus relationships in mycobacterium, nocardia and actinomyces. Juhasz SE, Plummer G (eds.). Charles C. Thomas, Springfield, pp80-102, 1970.

22. David HL, Clavel S, Clement F and Moniz-Pereira J: Effects of antituberculosis and antileprosy drugs on mycobacteriophage D29 growth. Antimicrob Agents Chemother 18: 357-359, 1980.

23. Wilson SM, al-Suwaidi Z, McNerney R, et al: Evaluation of a new rapid bacteriophage-based method for the drug susceptibility testing of Mycobacterium tuberculosis. Nat Med 3: 465-468, 1997.

24. McNerney R, Wilson SM, Sidhu AM, et al: Inactivation of mycobacteriophage D29 using ferrous ammonium sulphate as a tool for the detection of viable Mycobacterium smegmatis and M. tuberculosis. Res Microbiol 149: 487-495, 1998.

25. Mole RJ and Maskell TW: Phage as a diagnostic - the use of phage in TB diagnosis. J Chem Technol Biotechnol 76: 683-688, 2001.

26. Seaman T, Trollip A, Mole R, et al: The use of a novel phage-based technology as a practical tool for the diagnosis of tuberculosis in Africa. Afr J Biotechnol 2: 40-45, 2003.

27. Marei AM, El-Behedy EM, Mohtady HA and Afify AF: Evaluation of a rapid bacteriophage-based method for the detection of Mycobacterium tuberculosis in clinical samples. J Med Microbiol 52: 331-335, 2003.

28. Muzaffar R, Batool S, Aziz F, et al: Evaluation of the FASTPlaqueTB assay for direct detection of Mycobacterium tuberculosis in sputum specimens. Int J Tuberc Lung Dis 6: 635-640, 2002.

29. Albert H, Trollip AP, Mole RJ, et al: Rapid indication of multidrug-resistant tuberculosis from liquid cultures using FASTPlaqueTB-RIF ${ }^{\mathrm{TM}}$, a manual phage-based test. Int J Tuberc Lung Dis 6: 523-528, 2002.

30. Alcaide F, Galí N, Domínguez J, et al: Usefulness of a new mycobacteriophage-based technique for rapid diagnosis of pulmonary tuberculosis. J Clin Microbiol 41: 2867-2871, 2003.

31. Kalantri S, Pai M, Pascopella L, et al: Bacteriophage-based tests for the detection of Mycobacterium tuberculosis in clinical specimens: a systematic review and meta-analysis. BMC Infect Dis 5: 59, 2005.

32. Foundation for Innovative New Diagnostics (FIND). FIND interrupts demonstration projects using phage-based assays for detection of rifampin resistance. Geneva, Switzerland: FIND, 3 October 2007. http://www.finddiagnostics. org/media/news/index.jsp?year=2007\&domain. Accessed 16 May, 2014

33. Sarkis GJ, Jacobs WR Jr and Hatfull GF: L5 luciferase reporter mycobacteriophages: a sensitive tool for the detection and assay of live mycobacteria. Mol Microbiol 15: 1055-1067, 1995.

34. Pearson RE, Jurgensen S, Sarkis GJ, et al: Construction of D29 shuttle phasmids and luciferase reporter phages for detection of mycobacteria. Gene 183: 129-136, 1996.

35. Kumar V, Loganathan P, Sivaramakrishnan G, et al: Characterization of temperate phage Che12 and construction of a new tool for diagnosis of tuberculosis. Tuberculosis (Edinb) 88: 616-623, 2008

36. Carrière $\mathrm{C}$, Riska $\mathrm{PF}$, Zimhony $\mathrm{O}$, et al: Conditionally replicating luciferase reporter phages: improved sensitivity for rapid detection and assessment of drug susceptibility of Mycobacterium tuberculosis. J Clin Microbiol 35: 3232-3239, 1997.

37. Riska PF, Jacobs WR Jr, Bloom BR, et al: Specific identification of $M$. tuberculosis with the luciferase reporter mycobacteriophage: use of p-nitro-alpha-acetylamino-beta-hydroxy propiophenone. J Clin Microbiol 35: 3225-3231, 1997.

38. Dye C, Scheele S, Dolin P, Pathania V and Raviglione MC: Consensus statement. Global burden of tuberculosis: Estimated incidence, prevalence, and mortality by country. WHO Global Surveillance and Monitoring Project. JAMA 282: 677-686, 1999. 
39. Dusthackeer A, Kumar V, Subbian S, et al: Construction and evaluation of luciferase reporter phages for the detection of active and non-replicating tubercle bacilli. J Microbiol Methods 73: 18-25, 2008.

40. Dusthackeer VN, Balaji S, Gomathi NS, et al: Diagnostic luciferase reporter phage assay for active and non-replicating persistors to detect tubercle bacilli from sputum samples. Clin Microbiol Infect 18: 492-496, 2012.

41. Banaiee N, January V, Barthus C, et al: Evaluation of a semi-automated reporter phage assay for susceptibility testing of Mycobacterium tuberculosis isolates in South Africa. Tuberculosis (Edinb) 88: 64-68, 2008.
42. Minion J and Pai M: Bacteriophage assays for rifampicin resistance detection in Mycobacterium tuberculosis: updated meta-analysis. Int J Tuberc Lung Dis 14: 941-951, 2010.

43. Rondón L, Piuri M, Jacobs WR Jr, et al: Evaluation of fluoromycobacteriophages for detecting drug resistance in Mycobacterium tuberculosis. J Clin Microbiol 49: 1838-1842, 2011.

44. da Silva JL, Piuri M, Broussard G, et al: Application of BRED technology to construct recombinant D29 reporter phage expressing EGFP. FEMS Microbiol Lett 344: 166-172, 2013.

45. Piuri M, Rondón L, Urdániz E and Hatfull GF: Generation of affinity-tagged fluoromycobacteriophages by mixed assembly of phage capsids. Appl Environ Microbiol 79: 5608-5615, 2013. 